\title{
Comment on Numerical Deficiencies in the Paper by Al-Odat and Al-Hussien (Int. J. Thermophys. 29, 1523 (2008))
}

\section{Lewandowska}

Received: 6 December 2008 / Accepted: 23 July 2010 / Published online: 10 August 2010 (C) The Author(s) 2010. This article is published with open access at Springerlink.com

\begin{abstract}
Recently, Al-Odat and Al-Hussien presented consideration of the critical energy of a composite superconductor in this journal. Various numerical and other deficiencies have been identified in this paper. These problems are discussed and clarified in this Comment.
\end{abstract}

Keywords Composite superconductors · Critical energy · Hyperbolic equation of heat conduction - Thermal stability

\section{Introduction}

Recently, Al-Odat and Al-Hussien [1] have studied the critical energy of a cooled composite superconductor using the one-dimensional hyperbolic model of heat conduction, which takes into account the finite speed of heat propagation. The superconductor under consideration is subjected to a local heat disturbance of finite length and duration. These conditions cause temperature-dependent global ohmic heat generation. Critical energies of the conductor are calculated by analysis of the maximum temperature in the center of the normal zone.

It turns out that the paper [1] overlaps to some extent with an earlier paper co-authored by the present author [2], which dealt with the case of an uncooled composite superconductor. Careful analysis and comparison of [1] and [2] reveal numerous cases of numerical and other deficiencies in [1]. The aim of this Comment is to clarify and correct these deficiencies. Errors in the formulation and analysis of the problem in

\footnotetext{
M. Lewandowska $(\varangle)$

Institute of Physics, West Pomeranian University of Technology, Szczecin, Al. Piastów 48, 70-311 Szczecin, Poland

e-mail: monika.lewandowska@zut.edu.pl
} 
question are discussed in Sect. 2. Corrections to the numerical calculations and figures are presented in Sect. 3. A summary is given in Sect. 4.

\section{Problem Formulation and Analysis Deficiencies}

The model and the method of analysis in [1] are similar to those presented in [2]. From a mathematical point of view, the problem solved in [1] (Eqs. 12-16 therein) is essentially the same as the problem discussed in [2] (Eqs. 9-13 therein). Consequently, the solution of the problem in [1] is the same as in [2]. The only difference is that a constant $\alpha=G /\left(1+\theta_{\mathrm{cs}}\right)$ in the heat conduction equation, Eq. 6 in [2], is replaced by $\left[G /\left(1+\theta_{\mathrm{c}}\right)-B i\right]$ in Eq. 10 in [1]. Note that the dimensionless current sharing temperature $\theta_{\mathrm{cs}}$, i.e., the dimensionless critical temperature at a given current, given in Eq. 10 in [1] and in further equations (Eqs. 12, 13, 17, 18, 20-22, 24a, 24b, 26-28a in [1]), is inappropriately referred to by the symbol $\theta_{c}$, i.e., the dimensionless critical temperature at $I=0$.

The critical energy of the conductor is determined in [1] by analysis of the time evolution of the maximum temperature in the normal zone: $\theta_{1}(0, \tau)$. It is assumed that the critical energy of the conductor is equal to the energy of the disturbance if the minimum value of $\theta_{1}(0, \tau)$ for $\tau \geq \tau_{i}$, i.e., after switching off the heat disturbance, is equal to the current sharing temperature $\theta_{\mathrm{cs}}$. This method was originally proposed by Malinowski in [3] and utilized in [2].

We have identified some errors in the formulation of the problem, as well as in the solution, as indicated below.

- The authors start their study with the phrase: "Consider a very long composite superconductor of diameter $(d)$ carrying an electrical current perpendicular to the $x-y$ plane," whereas in a later part they use a one-dimensional model with the $x$ coordinate directed along the conductor.

- It is stated in [1]: "Due to the symmetry of the normal zone, the analysis is limited to the half of the zone, i.e., to the domain that lies within $0 \geq x \geq l$." In fact, the analysis is limited to the region, $x \geq 0$. This is due to the location of the origin of the $x$ coordinate in the center of the region subjected to the local heat disturbance and the resulting spatial symmetry of the normal zone.

- Several errors of various nature are identified by us in equations. The original (O) equations taken from [1] and the corrected (C) ones, as well as some explanations are given below. For conciseness the following abbreviations are introduced:

$$
\begin{aligned}
\gamma & =\frac{G}{1-\theta_{\mathrm{cs}}}-B i, \\
\gamma^{\prime} & =\frac{G}{1-\theta_{\mathrm{c}}}-B i, \\
\gamma^{\prime \prime} & =-\frac{G}{1-\theta_{\mathrm{c}}}-B i .
\end{aligned}
$$


i Energy balance equation:

$$
\begin{aligned}
& \text { O: } C \frac{\partial T(x, t)}{\partial t}=k \nabla \cdot \mathbf{q}-\frac{P}{A}\left[h\left(T-T_{0}\right)+g(T)+D(x, t)\right] \text { Eq. } 1 \text { in [1] } \\
& \text { C: } C \frac{\partial T}{\partial t}=-k \frac{\partial q}{\partial x}-\frac{P}{A}\left[h\left(T-T_{0}\right)-g(T)-D(x, t)\right]
\end{aligned}
$$

This equation should be consequently written either in a vector or in a one-dimensional form. The heat conduction term (the 1st term) in the r.h.s. should be negative, whereas the Joule heating and thermal disturbance terms (the 3rd and 4th terms, respectively) should be positive.

ii Boundary and initial conditions in dimensional form:

$$
\begin{gathered}
\text { O: } T(x, 0)=T_{0} \quad \frac{\partial T(x, y, 0)}{\partial t}=\dot{T}_{i} \quad \text { Eqs. } 7 \text { in [1] } \\
T(-l, t)=T(-l, t)=T_{0} \quad \text { Eq. } 8 \text { in [1] } \\
\begin{array}{c}
\text { C: } T_{1}(x, 0)=T_{0} \quad T_{2}(x, 0)=T_{0} \\
\frac{\partial T_{1}}{\partial t}(x, 0)=\frac{E}{2 A C l t_{i}} \quad \frac{\partial T_{2}}{\partial t}(x, 0)=0 \\
\frac{\partial T_{1}}{\partial x}(0, t)=0 \quad T_{2}(\infty, t)=T_{0} \\
T_{1}(l, t)=T_{2}(l, t) \quad \frac{\partial T_{1}}{\partial x}(l, t)=\frac{\partial T_{2}}{\partial x}(l, t)
\end{array}
\end{gathered}
$$

The second initial condition in Eqs. 7 in [1] is unclear, because the constant $\dot{T}_{i}$ is undefined. The boundary condition, Eq. 8 in [1], makes no sense-apart from the misprint ( $-l$ should be replaced by $l$ ), the assumption that the temperature at the boundary of the region heated by the local source is constant is unrealistic and inconsistent with what is written later. Some boundary conditions are missing. For consistency with further analysis, the boundary and initial conditions should be formulated for $T_{1}$ and $T_{2}$ separately, where $T_{1}$ is the temperature in the region subjected to the heat disturbance $(0 \leq x \leq l)$ and $T_{2}$ is the temperature in the rest of the conductor $(x>l)$. The initial conditions, Eqs. C4, result from the assumption that there is no heat flow at the initial moment $(q(x, 0)=0)$ and from the energy balance equation, Eq. C2. The first boundary condition in Eqs. C5 is based on the assumption $q(0, t)=0$, which results from the symmetry of the normal zone. The boundary conditions, Eqs. C6, are based on the assumption that the temperature and the heat flux are continuous at the boundary of the region subjected to the local heat source, i.e., at $x=l$.

iii Dimensionless boundary conditions:

$$
\begin{array}{ll}
\text { O: } \frac{\partial \theta_{1}(0, \tau)}{\partial \tau}=0 & \frac{\partial \theta_{1}(1, \tau)}{\partial \tau}=\frac{\partial \theta_{2}(1, \tau)}{\partial \tau} \quad \text { Eq. } 15 \text { in [1] } \\
\text { C: } \frac{\partial \theta_{1}}{\partial \xi}(0, \tau)=0 & \frac{\partial \theta_{1}}{\partial \xi}(1, \tau)=\frac{\partial \theta_{2}}{\partial \xi}(1, \tau)
\end{array}
$$


iv Solution of the problem in question:

$\mathrm{O}: \bar{\theta}_{1}(\xi, s)=\frac{\varphi \bar{u}(s)}{2 \tau_{i}\left(s-\gamma^{\prime}\right)}-\{2-\exp [-(1-\xi) \sqrt{\psi(s)}]$

$-\exp [-(1+\xi) \sqrt{\psi(s)}]\} \quad$ Eq. 20 in [1]

$\mathrm{C}: \bar{\theta}_{1}(\xi, s)=\frac{\varphi \bar{u}(s)}{2 \tau_{i}(s-\gamma)} \cdot\{2-\exp [-(1-\xi) \sqrt{\psi(s)}]$

$-\exp [-(1+\xi) \sqrt{\psi(s)}]\}$

O: $\bar{\theta}_{2}(\xi, s)=\frac{\varphi \bar{u}(s)}{2 \tau_{i}\left(s-\gamma^{\prime}\right)}-\{\exp [(1-\xi) \sqrt{\psi(s)}]$

$-\exp [-(1+\xi) \sqrt{\psi(s)}]\} \quad$ Eq. 21 in [1]

$\mathrm{C}: \bar{\theta}_{2}(\xi, s)=\frac{\varphi \bar{u}(s)}{2 \tau_{i}(s-\gamma)} \cdot\{\exp [(1-\xi) \sqrt{\psi(s)}]$

$$
-\exp [-(1+\xi) \sqrt{\psi(s)}]\}
$$

O: $\bar{\theta}_{1}(0, s)=\frac{\varphi \bar{u}(s)}{2 \tau_{i}\left(s-\gamma^{\prime}\right)}-\{1-\exp [\sqrt{\psi(s)}]\} \quad$ Eq. 22 in [1]

$\mathrm{C}: \bar{\theta}_{1}(0, s)=\frac{\varphi \bar{u}(s)}{\tau_{i}(s-\gamma)} \cdot\{1-\exp [-\sqrt{\psi(s)}]\}$

O: $\psi_{1}(\tau)=\frac{1}{\left(s-\gamma^{\prime}\right)}-\exp \left[\gamma^{\prime} \tau\right]\left\{1-\exp \left[\gamma^{\prime} \tau_{q}\right]\right\} \quad$ Eq. 24a in [1]

$\mathrm{C}: \psi_{1}(\tau)=\frac{1}{\gamma} \cdot \exp (\gamma \tau)\left[1-\exp \left(-\gamma \tau_{a}\right)\right], \quad$ where $\tau_{a}=\min \left(\tau, \tau_{i}\right)$

$\mathrm{O}: \psi_{2}(\tau)=\exp \left(\frac{\gamma^{\prime}\left(\tau_{q}-1\right)}{2 \sqrt{\tau_{q}}}\right) \psi_{1}\left(\tau-\sqrt{\tau_{q}}\right)+\left(\frac{\gamma^{\prime}\left(\tau_{q}-1\right)}{2 \sqrt{\tau_{q}}}\right)$

$$
\times \int_{\sqrt{\tau_{q}}}^{\tau_{a}} \psi_{1}(\tau-y)\left(\exp \left(\gamma^{\prime \prime} \tau_{q}\right)-1\right) \frac{y}{2 \tau_{q}}
$$

$$
\times I_{1}\left(\gamma^{\prime \prime}\right) \tau_{q} \sqrt{\left(y^{2}-\tau_{q}\right)} \text { Eq. 24b in [1] }
$$

$\mathrm{C}: \psi_{2}(\tau)=\exp \left(\frac{\gamma \tau_{q}-1}{2 \sqrt{\tau_{q}}}\right) \psi_{1}\left(\tau-\sqrt{\tau_{q}}\right)+\frac{\gamma \tau_{q}+1}{2 \sqrt{\tau_{q}}}$

$$
\begin{aligned}
& \times \int_{\sqrt{\tau_{q}}}^{\tau} \psi_{1}(\tau-y) \exp \left[\frac{\left(\gamma \tau_{q}-1\right) y}{2 \tau_{q}}\right] I_{1}\left[\frac{\gamma \tau_{q}+1}{2 \tau_{q}} \sqrt{y^{2}-\tau_{q}}\right] \\
& \times \frac{1}{\sqrt{y^{2}-\tau_{q}}} \mathrm{~d} y
\end{aligned}
$$




$$
\begin{aligned}
& \text { O: } \theta_{1}(0, \tau)=\frac{\varphi}{\tau_{i}} \int_{0}^{\tau_{a}}-\exp \left[\gamma^{\prime}(\tau-y)\right] \operatorname{erf}[0.5 \sqrt{\tau-y}] \mathrm{d} y \quad \text { Eq. } 27 \text { in [1] } \\
& \text { C: } \theta_{1}(0, \tau)=\frac{\varphi}{\tau_{i}} \int_{0}^{\tau_{a}} \exp [\gamma(\tau-y)] \operatorname{erf}\left(\frac{0.5}{\sqrt{\tau-y}}\right) \mathrm{d} y \\
& \text { O: } \varepsilon_{p}=\frac{\tau_{i} \theta_{\mathrm{cs}}}{\int_{0}^{\tau_{i}} \exp \left(\gamma^{\prime}\right)\left[\left(\tau_{\mathrm{m}}-y\right) \operatorname{erf}\left[0.5\left(\tau_{\mathrm{m}}-y\right)^{-0.5}\right]\right] \mathrm{d} y} \quad \text { Eq. 28a in [1] } \\
& \text { C: } \varepsilon_{p}=\frac{\tau_{i} \theta_{\mathrm{cs}}}{\int_{0}^{\tau_{i}} \exp \left[\gamma\left(\tau_{\mathrm{m}}-y\right)\right] \operatorname{erf}\left[0.5\left(\tau_{\mathrm{m}}-y\right)^{-0.5}\right] \mathrm{d} y}
\end{aligned}
$$

\section{Corrections to the Numerical Calculations and Figures}

Examples of the variation of the $\theta_{1}(0, \tau)$ with time for various values of the dimensionless ohmic heat generation, $G$, which were supposed to illustrate the method of assessment of the critical energy, are presented in Fig. 1 in [1]. However, captions associated with that figure are confusing.

- The value of parameter $\theta_{\mathrm{cs}}$ is not given. Hence, it is impossible to check if the disturbance energy, $\varphi=0.5$, is really equal to the critical energy for $G=1$ and one cannot be sure if the method of assessment of the critical energy is applied properly. One may only guess that probably $\theta_{\mathrm{cs}} \approx 4$, which is the minimum value

Fig. 1 Maximum dimensionless temperature in the normal zone versus dimensionless time for the hyperbolic ( $\tau_{q}=0.1$, dashed lines) and the related parabolic model ( $\tau_{q}=0$, solid lines) at $\theta_{\mathrm{cs}}=4$, $\tau_{i}=0.2, \varphi=0.5, B i=0.1$

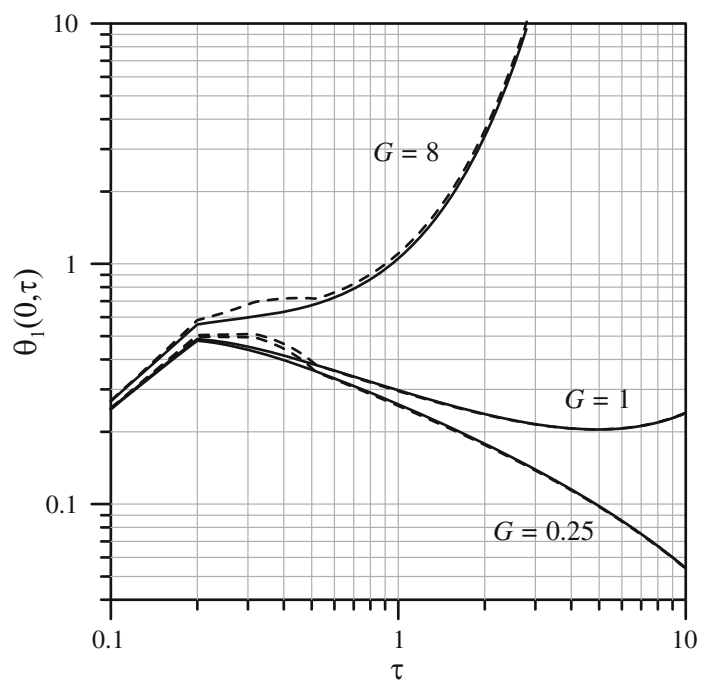


Fig. 2 Variance of the maximum dimensionless temperature in the normal zone with time for the hyperbolic ( $\tau_{q}=0.1$, dashed lines $)$ and the related parabolic model $\left(\tau_{q}=0\right.$, solid lines) at $\theta_{\mathrm{cs}}=0.3$, $\tau_{i}=0.1, \varphi=0.447, B i=0.1$

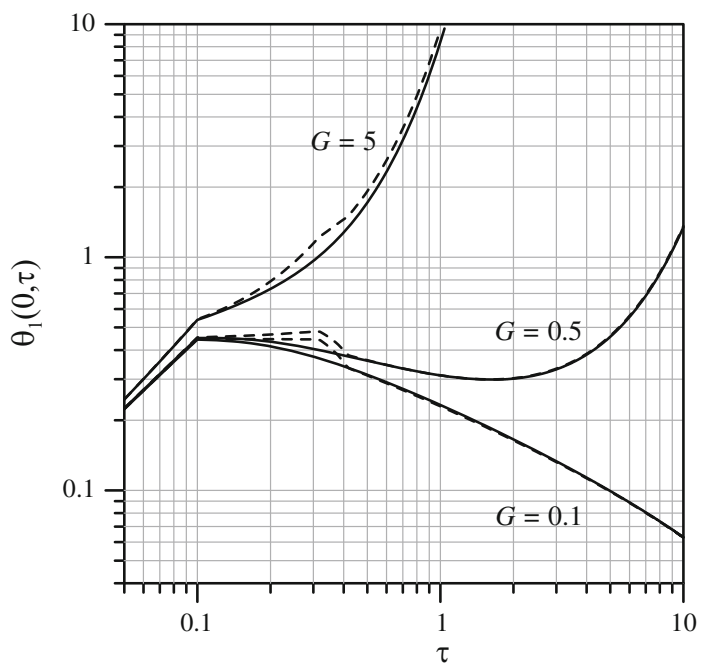

of $\theta_{1}(0, \tau)$ for $G=1$ and $\tau \geq \tau_{i}$. This assertion will be checked further by our calculations.

- It is stated in the caption for Fig. 2 in [1] that the duration of the heat disturbance, $\tau_{i}$, is equal to 0 . It is seen from the shape of the curves that $\tau_{i} \approx 0.2$.

- The lowest value of $G$ indicated in the figure is 0.25 which disagrees with the value $G=0.2$ stated in the text above Fig. 2 in [1].

We have tried to reproduce the results presented in Fig. 2 in [1]. For this purpose, the hyperbolic solutions are obtained according to Eq. 23 in [1] as

$$
\theta_{1}(0, \tau)= \begin{cases}\frac{\varphi}{\tau_{i}} \psi_{1}(\tau) & \text { for } \tau<\sqrt{\tau_{q}} \\ \frac{\varphi}{\tau_{i}}\left[\psi_{1}(\tau)-\psi_{2}(\tau)\right] & \text { for } \tau \geq \sqrt{\tau_{q}}\end{cases}
$$

using the corrected expressions for $\psi_{1}$ and $\psi_{2}$, i.e., Eqs. C11 and C12, respectively. The related parabolic solutions were obtained using Eq. C13. The results of our calculations for $\theta_{\mathrm{cs}}=4, \tau_{i}=0.2, \tau_{q}=0$ and $0.1, \varphi=0.5, B i=0.1$, and three values of the dimensionless ohmic heat generation $G=0.25,1$, and 8 are presented in Fig. 1. These values of parameters are assumed to be the same as those used in Fig. 2 in [1]. However, our calculations yield significant quantitative differences between the solutions presented in Figs. 1 and 2 in [1]. Importantly, the dimensionless temperatures obtained by us are much lower and the dimensionless energy of disturbance $\varphi=0.5$ is lower than the dimensionless critical energy for both $G=0.25$ and $G=1$. Thus, there are serious doubts if the calculations presented in [1] were performed correctly. The correctness of the analytical hyperbolic and parabolic solutions presented in [2] and [3] had been carefully tested by comparison with the respective numerical solutions, and the critical energies determined in [2] and [3] were found in good agreement with experimental data taken from [4]. 
Fig. 3 Dependence of the dimensionless critical energy on the Biot number for various dimensionless ohmic heat generation and relaxation times at $\tau_{i}=0.25, \theta_{\mathrm{cs}}=0.3$, $\tau_{q}=0.2$ (dashed lines) and $\tau_{q}=0$ (parabolic model, solid lines)

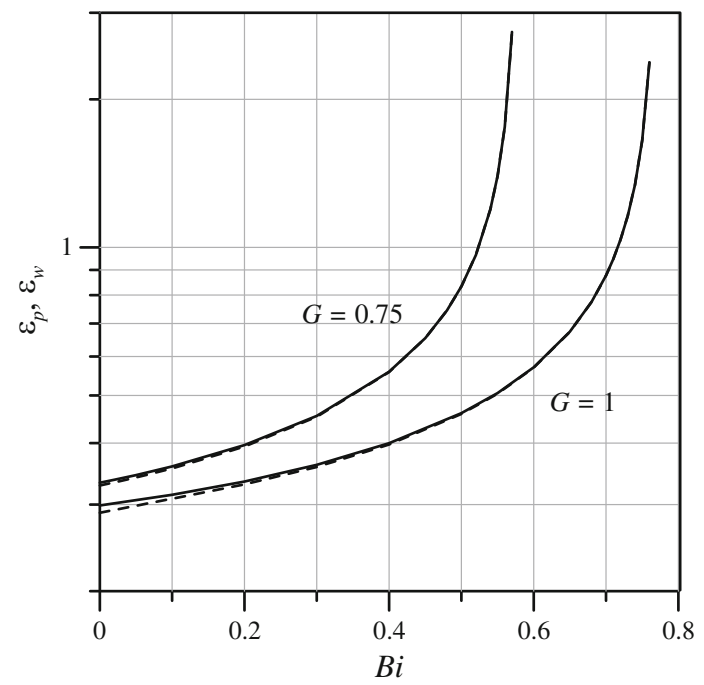

To illustrate correctly the method of determining the critical energy and the influence of cooling on its value, we performed the calculations for $\theta_{\mathrm{cs}}=0.3, \tau_{i}=0.1$, $\tau_{q}=0$ and $0.1, \varphi=0.447, B i=0.1$, and $G=0.1,0.5$, and 5, and the results are shown in Fig. 2. The same values of parameters $\theta_{\mathrm{cs}}, \tau_{i}, \tau_{q}$, and $G$, and $\varphi=0.3931$, used in Fig. 1 in [2], were adopted by us for the uncooled conductor $(B i=0)$. It is seen in Fig. 2 that the energy of disturbance $\varphi$ is smaller than the critical energy for $G=0.1$; for $G=0.5, \varphi$ is equal to the critical energy; and for $G=5, \varphi$ is greater than the critical energy. The critical energy of the uncooled conductor is lower than the critical energy of the respective cooled conductor as expected.

In Fig. 3, the dependence of the dimensionless critical energy obtained from the parabolic model, $\varepsilon_{p}$, and from the corresponding hyperbolic model, $\varepsilon_{w}$, on the Biot number is shown. It is seen that the critical energy increases rapidly with the Biot number and reaches infinity at $B i=G /\left(1+\theta_{\mathrm{cs}}\right)$. Infinite value of the critical energy means that the cooling is always larger than the heat dissipation and generation, so the conductor will cool down after every disturbance. A conductor fulfilling this condition is fully stable.

The critical energies calculated using the hyperbolic model are always lower than the respective values predicted by the parabolic model, which has been discussed in [2]. However, for the considered range of parameters, the differences between the $\varepsilon_{p}$ and $\varepsilon_{w}$ are small (less than $3.5 \%$ at $B i=0$ ) and they decrease with $B i$. This effect is clearly seen in Fig. 4, which presents the percentage differences between the critical energies $\varepsilon_{p}$ and $\varepsilon_{w}$, defined as $\Delta \varepsilon=100\left(\varepsilon_{p}-\varepsilon_{w}\right) / \varepsilon_{p}$ (in $\%$ ), as a function of the Biot number. The higher is the value of $B i$, the more heat is transferred to the coolant and the less heat is transported along the conductor by conduction, so the influence of the used model of the heat conduction on the critical energy becomes less significant. 
Fig. 4 Percentage differences between critical energies calculated from the parabolic and the related hyperbolic models versus Biot number for various dimensionless ohmic heat generation and relaxation times at $\tau_{i}=0.25, \theta_{\mathrm{cs}}=0.3$, $\tau_{q}=0.2$ (dashed lines), $\tau_{q}=0.1$ (dashed-dotted lines), and $\tau_{q}=0.05$ (solid lines)

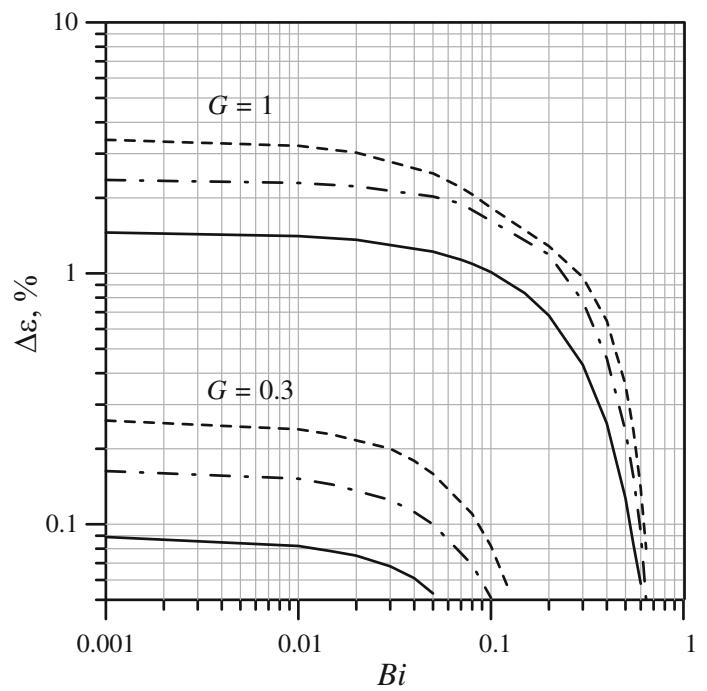

\section{Summary}

Some equations and numerical results presented in Ref. [1] turn out to be incorrect. Since the analytical solutions are often used for testing more complex numerical models, it is essential to correct and clarify the identified deficiencies. The errors identified in the formulation of the problem and in the solutions have been discussed and corrected. The corrected analysis of the dependence of the critical energy on cooling has been presented.

Acknowledgments The author is greatly indebted to Profs. L. Malinowski and Cz. Rudowicz for their reading of the manuscript and valuable comments.

Open Access This article is distributed under the terms of the Creative Commons Attribution Noncommercial License which permits any noncommercial use, distribution, and reproduction in any medium, provided the original author(s) and source are credited.

\section{References}

1. M.Q. Al-Odat, F.M. Al-Hussien, Int. J. Thermophys. 29, 1523 (2008)

2. M. Lewandowska, L. Malinowski, Cryogenics 41, 267 (2001)

3. L. Malinowski, Cryogenics 39, 311 (1999)

4. C. Schmidt, Cryogenics 18, 605 (1978) 